\title{
Perception of Taichi diagram (a) Birth, aging, sickness, and death
}

\author{
SHI Jian-ming \\ Shanghai tongji hospital (tongji hospital of tongji university), Traditional Chinese Medicine \\ department, Shanghai, China
}

EMAIL: alexcellent@hotmail.com

Keywords: Taichi diagram; Yin; Yang; birth; aging; sickness; death

\begin{abstract}
Ebb and flow of Yin and Yang in Taichi diagram is the interpretation of birth, aging, sickness and death. This article paper clarifies what is the birth, aging, sickness, and death, from Taichi diagram, Yin and Yang theory. The whole process of birth, aging, sickness, and death is: Yin gradually enlarges flowing down continuously in lower part of human body, Yang gradually enlarges rising too hyperactively continuously in the upper part of human body. Until the final separation ,separating and severing between Yin and Yang. On the other hand, the number of Yin flowing down in the lower part of humam body is equal to the number of Yang rising too hyperactively in the upper part of human body. They are equivalently corresponding between them, interacting at the same time between them, reciprocally causing between them.
\end{abstract}

\section{Perception:}

Taichi diagram is used to explain the inherent law of everything in the vast universe. Simple and all-inclusive. The main purpose is to explain life. Explaining life mainly refers to the interpretation of the natural process of life: birth, aging, sickness, and death.

As long as there is birth for any life on the earth, then the birth, aging, sickness, and death is its inevitable destination. (Natural death) Taichi diagram is the explanation to the most fundamental question in life.

\section{The layer 1 meaning of Taichi diagram :}
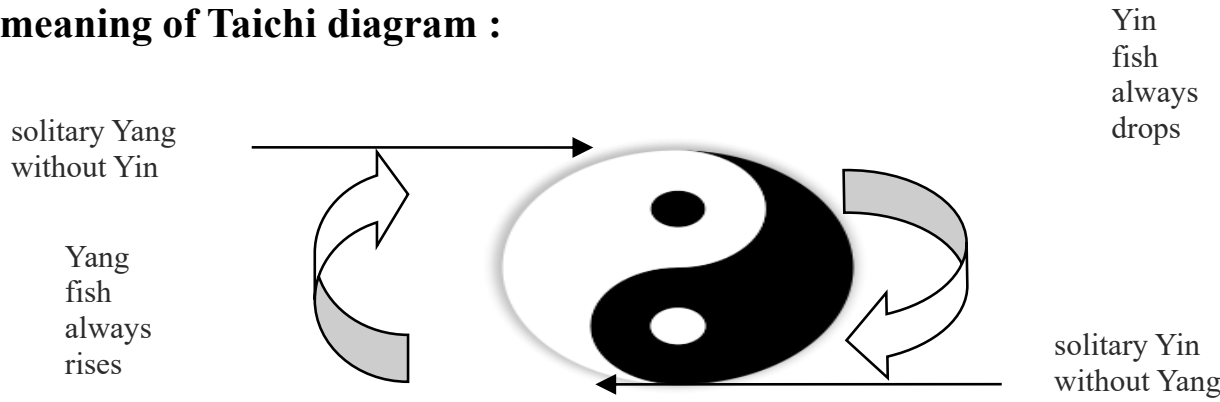

without Yang

Figure1 The layer 1 meaning of Taichi diagram

2.1 Yang always rises and Yin always drops (Yang fish always rises and Yin fish always drops), (Figure 1) it is determined by the Yin and Yang inherent attribute. ${ }^{[1]}$

2.2 Yang on the left rises and Yin on the right drops ${ }^{[2]}$.

2.3 Yang on the left, from one Yang, to two Yang, then to three Yang, taiYang, rising too hyperactively in the upper; Yin on the right, from one Yin, to two Yin ,then to three Yin , taiYin ${ }^{[3]}$, flowing down in the lower. Three Yang, TaiYang is already the largest Yang; Three Yin, TaiYin is already the largest Yin. According to Yin and Yang theory of $<$ Neijing $>$, According to Taichi diagram ,we can observe obviously :while TaiYang in the upper, there is already solitary Yang without Yin ; while TaiYin in the lower, there is already solitary Yin without Yang.

$<$ Suwen.shengqitongtianlun> said: "If Yin and Yang separate from each other, essential qi will be 
exhausted."[4] Separating and Severing between Yin and Yang means death, It is the time of death ......So, when Yang fish in Taichi diagram is taiYang, the largest Yang, solitary Yang without Yin in the upper; the Yin fish in Taichi diagram is taiYin, the largest Yin, solitary Yin without Yang in the lower, It does indicate the state of separating and severing between Yin and Yang. It does point out what is the death. (Figure 1)

\section{The layer 2 meaning of Taichi diagram}

one Yin is born - birth

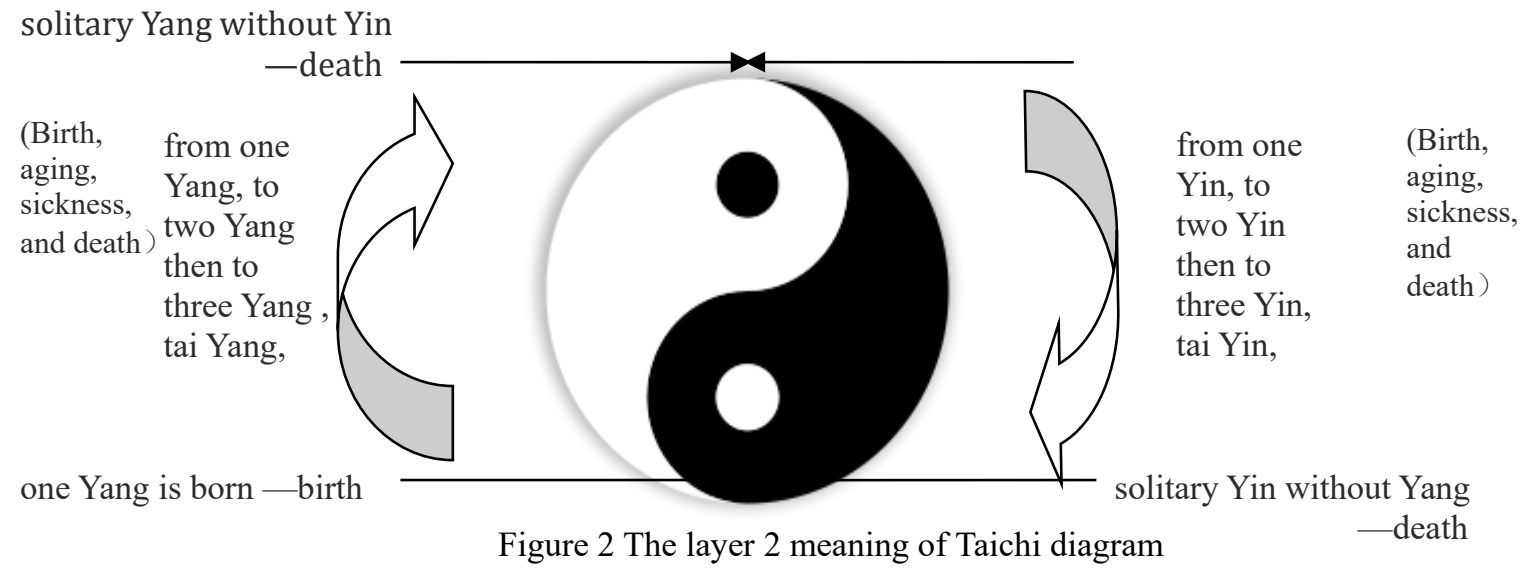

3.1 Between birth and death, that is, the whole process of life---- birth, aging, sickness and death. That is to say: the whole process of birth, aging, sickness and death is: A, Yin gradually enlarges flowing down continuously in lower part of human body (from one Yin, to two Yin, then to three Yin, tai Yin, solitary Yin without Yang) B, Yang gradually enlarges rising too hyperactively continuously in the upper part of human body (from one Yang, to two Yang, then to three Yang ,tai Yang, solitary Yang without Yin). C, Until the final separation, Separating and Severing between Yin and Yang. D, On the other hand, the number of Yin flowing down in the lower part of human body is equal to the number of Yang rising too hyperactively in the upper part of human body. they are equivalently corresponding between them, interacting at the same time between them, reciprocally causing between them. (Figure 2)

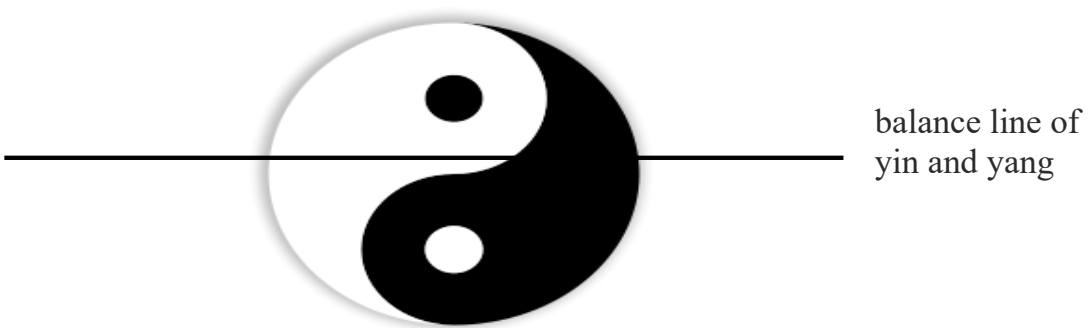

Figure 3 balance line of yin and yang

3.2 In the middle of the Taichi diagram, Drawing a line left and right direction. Taichi diagram is just divided into equal two parts too. This line is drawed by myself. It has not been recorded yet. The name might be the balance line of Yin and Yang. Just as its name implies, also according to the above: when the line passes by, there are half Yin and half Yang (Yin is equal to Yang) in human body and in all lives at that time(balance of Yin and Yang). Then if yang of the human body rises and Yin of human body drops by following their inherent attribute, it is the time of the peak of physiological function in human body, when the line passes by. after this line, it indicates that the loss starts, after this line, it indicates the road to the aging and sickness. after this line, it indicates the road to death. Corresponding to human growth and development in TCM theory, I think it is the time that Tiankui arrives ${ }^{[1]}$, when the balance line of Yin and Yang passes by. (Figure 3) 


\section{The layer 3 meaning of Taichi diagram}

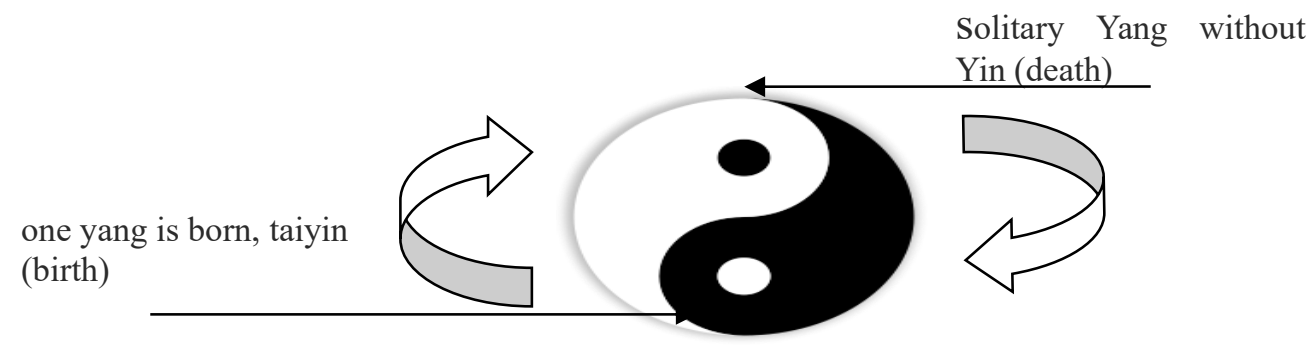

Figure 4 upper part of life

4.1 In the process of life : birth, aging, sickness, and death, in Taichi diagram, From the point of view of Yang fish on the left, From the point of view of upper part of human body-- the Yang aspect of human body, from one Yang, to two Yang, then to three Yang, taiYang, solitary Yang without Yin in the upper part of human body. At the same time, in the upper part of human body, contrasting simultaneously, one ebbing and another flowing, in Yin aspect, from solitary Yin without Yang, taiYin, three Yin, to two Yin, then to one Yin. (Figure 4)

solitary Yin without

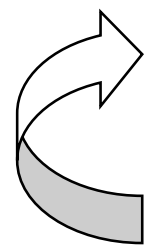

Yang (death)
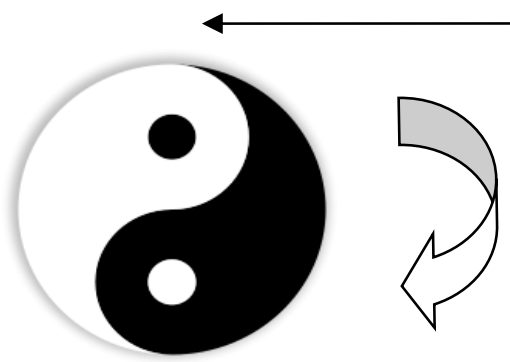

one Yin is born ,taiYang (birth)

Figure 5 Lower part of life

4.2 In the process of life : birth, aging, sickness, and death, in Taichi diagram,

From the point of view of Yin fish on the right, From the point of view of lower part of human body-- the Yin aspect of human body, from one Yin, to twoYin, then to threeYin, taiYin, solitary Yin without Yang in the lower part of human body. At the same time, in the lower part of human body, contrasting simultaneously, one ebbing and another flowing, in Yang aspect, from solitary Yang without Yin, taiYang, three Yang, to two Yang, then to one Yang. (Figure 5)

4.3 So we can see that: In the process of life : birth, aging, sickness, and death, the number of Yin flowing down in the lower part of human body is equal to the number of Yang rising too hyperactively in the upper part of human body. and the number of Yin saving in the upper part of human body is equal to the number of Yang saving in the lower part of human body. they are equivalently corresponding between them, interacting at the same time between them, reciprocally causing between them.

\section{Corresponding author:}

SHI Jian-ming(1971-),male,born in China. Shanghai tongji hospital affiliated to tongji university school of medicine, Traditional Chinese Medicine department 


\section{References :}

[1] CHENG-shide MENG-jingchun: NeiJing Lecture. SuWen.

YinYangyingxiangdalunpian. TheFifth.shanggutianzhenlunpian. TheFirst .ShangHai:Shanghai Science and Technology Press;1984:21 .176.

[2] ZHANG-jiebin: LeiChing.The.Second.YinYangLei . KuaiJi: ZhangShi; 1624

[3] WU-qian: YiZongJinJian.wuyunliuqiyaojue.keqisitianzaiquanjianqige.zhu . BeiJing: Qing dynasty; 1742

[4] LI-zhengji: Traditional Chinese medicine commonly used terms dictionary. BeiJing:China Traditional Chinese Medicine press ;2001:158 